\title{
Identification of novel prognostic markers of survival time in high-risk neuroblastoma using gene expression profiles
}

\author{
Abdulazeez Giwa ${ }^{1}$, Azeez Fatai ${ }^{2}$, Junaid Gamieldien ${ }^{1}$, Alan Christoffels ${ }^{1}$ and Hocine \\ Bendou ${ }^{1}$ \\ ${ }^{1}$ SAMRC Bioinformatics Unit, South African National Bioinformatics Institute, University of the Western Cape, Bellville, South \\ Africa \\ ${ }^{2}$ Department of Biochemistry, Lagos State University, Lagos, Nigeria \\ Correspondence to: Hocine Bendou, email: hocine@sanbi.ac.za
}

Keywords: neuroblastoma; differential gene expression; prognostic markers; machine learning; gene regulatory networks Received: July 02, $2020 \quad$ Accepted: October 27, $2020 \quad$ Published: November 17, 2020

Copyright: @ 2020 Giwa et al. This is an open access article distributed under the terms of the Creative Commons Attribution License (CC BY 3.0), which permits unrestricted use, distribution, and reproduction in any medium, provided the original author and source are credited.

\section{ABSTRACT}

Neuroblastoma is the most common extracranial solid tumor in childhood. Patients in high-risk group often have poor outcomes with low survival rates despite several treatment options. This study aimed to identify a genetic signature from gene expression profiles that can serve as prognostic indicators of survival time in patients of high-risk neuroblastoma, and that could be potential therapeutic targets. RNA-seq count data was downloaded from UCSC Xena browser and samples grouped into Short Survival (SS) and Long Survival (LS) groups. Differential gene expression (DGE) analysis, enrichment analyses, regulatory network analysis and machine learning (ML) prediction of survival group were performed. Forty differentially expressed genes (DEGs) were identified including genes involved in molecular function activities essential for tumor proliferation. DEGs used as features for prediction of survival groups included EVX2, NHLH2, PRSS12, POU6F2, HOXD10, MAPK15, RTL1, LGR5, CYP17A1, OR1OAB1P, MYH14, LRRTM3, GRIN3A, HS3ST5, CRYAB and NXPH3. An accuracy score of $82 \%$ was obtained by the ML classification models. SMIM28 was revealed to possibly have a role in tumor proliferation and aggressiveness. Our results indicate that these DEGs can serve as prognostic indicators of survival in high-risk neuroblastoma patients and will assist clinicians in making better therapeutic and patient management decisions.

\section{INTRODUCTION}

Neuroblastoma is the most common extracranial solid tumor in childhood accounting for approximately $15 \%$ of pediatric cancer death [1-3]. It develops anywhere along the sympathetic nervous system with $60 \%$ of the tumors occurring in the abdomen, commonly in the adrenal gland $[4,5]$.

Outcomes ranging from spontaneous regression to relentless progression despite extensive therapies indicate the heterogeneity of neuroblastoma [6]. The Children's Oncology Group (COG) classifies neuroblastoma patients into low, intermediate and high-risk groups. Patients classified in low-risk groups have good outcomes contrary to high-risk groups who present poor outcomes despite extensive therapies [4] and with a disproportionate number dying or suffering profound treatment related morbidities $[7,8]$. Tumors in high-risk neuroblastoma patients are often metastatic, resulting in survival rates of less than $50 \%[1]$.

Genomic studies associated high-risk neuroblastoma with mutations or alterations in a number of genes, such as ALK, ATRX and TERT [9-12]. Furthermore, genome wide association studies have revealed genetic markers, such as CASC15, LMO1, DUSP2 and BARD1 to be neuroblastoma susceptibility genes [13-21]. However, these genes do not provide information about patient survival. 
The objective of our study is to identify a genetic signature from gene expression data that can serve as prognostic indicators of survival time in high-risk neuroblastoma patients and that could be therapeutic targets in that patient category.

\section{RESULTS}

Querying the Xena TARGET dataset returned 20 and 12 SS and LS samples, respectively. Based on the gene expression levels in these samples, the edgeR filterByExpr function removed 35,873 low expressed genes and kept 24,610 genes for downstream analysis. The DGE analysis with DESeq2 identified 40 DEGs between the SS and LS groups, of which 21 genes were upregulated and 19 genes were downregulated. Table 1 shows information about the 40 DEGs.

The Gene Ontology (GO) Molecular Function enrichment analysis revealed that upregulated genes were mainly enriched in MAP kinase activity, retinol binding and RNA polymerase II activating transcription factor binding, as well as in other activities shown in (Figure 1A). No statistically significant results ( $p$-adjusted value $<0.05$ ) were obtained for the downregulated genes as well as for the other GO categories; Biological Process and Cellular
Component. In addition, the Disease Ontology enrichment analysis associated upregulated and downregulated genes with several genetic disorders; intellectual disability, cardiac dysfunction, bone development, impaired infertility and pulmonary dysfunction caused by diaphragm-associated abnormalities (Figure 1B and 1C).

Reconstruction of gene regulatory networks, using the GENIE3 algorithm, for the SS and LS samples respectively deduced 1,966,606 and 1,967,020 weighted interactions involving the DEGs. Applying a weight threshold value of 0.00251 resulted in 1018 and 650 DEG interactions for the SS and LS groups, respectively. The visualization of the 1018 DEG interactions using Cytoscape enabled the detection of 4 essential regulatory networks (Figure 2). The first network (Figure 2A) involves SMIM28, LGR5, PRSS12, EVX2, NHLH2 and HOXD10. All of these DEGs are upregulated, and the last three genes are transcription factors. The following network (Figure 2B) interconnect MAPK15, Lnc-ZNF814-1, EDIL3, NBAS and CYP17A1. The first two genes are upregulated and the last three genes are downregulated. Most of the DEGs in the third and last networks (Figure 2C and 2D) are downregulated with the exception of MEG9 and STRA6 which are upregulated. Interestingly, these interactions between the DEGs are
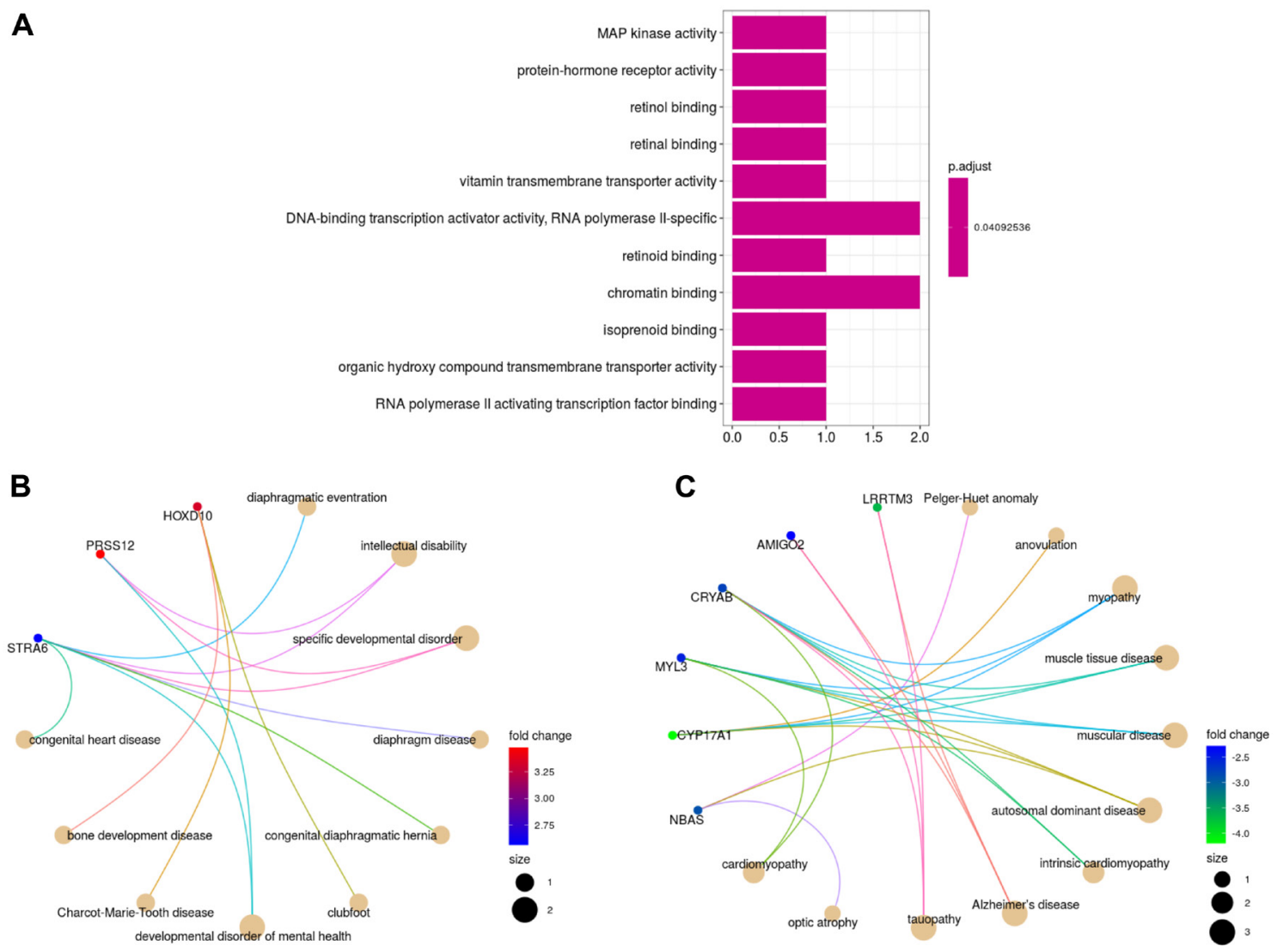

Figure 1: Gene and disease enrichment analyses. Molecular Function activities enriched by the upregulated DEGs (A). Network representations of enriched diseases for upregulated DEGs (B) and downregulated DEGs (C) (absolute value of fold change $>1.5$ ). The size of the circles represents the number of genes that enrich a disease. 
Table 1: Upregulated and downregulated DEGs in SS neuroblastoma samples compared to LS samples

\begin{tabular}{|c|c|c|c|c|}
\hline Symbol & Name & $\log 2 \mathrm{FC}$ & $p$-adj & Status \\
\hline SMIM28 & small integral membrane protein 28 & 5.4652 & 0.0146 & $\mathrm{Up}$ \\
\hline EVX2 & even-skipped homeobox 2 & 4.9304 & 0.0014 & Up \\
\hline NHLH2 & nescient helix-loop-helix 2 & 4.2404 & 0.0046 & Up \\
\hline PRSS12 & serine protease 12 & 3.4788 & 0.0168 & $\mathrm{Up}$ \\
\hline POU6F2 & POU class 6 homeobox 2 & 3.4534 & 0.0430 & Up \\
\hline HOXD10 & homeobox D10 & 3.3386 & 0.0357 & $\mathrm{Up}$ \\
\hline MAPK15 & mitogen-activated protein kinase 15 & 3.0939 & 0.0499 & Up \\
\hline RTL1 & retrotransposon Gag like 1 & 2.8233 & 0.0241 & $\mathrm{Up}$ \\
\hline LGR5 & leucine rich repeat containing $\mathrm{G}$ protein-coupled receptor 5 & 2.7453 & 0.0386 & Up \\
\hline DPY19L2P4 & DPY19L2 pseudogene 4 & 2.6439 & 0.0187 & Up \\
\hline STRA6 & signaling receptor and transporter of retinol STRA6 & 2.5625 & 0.0437 & Up \\
\hline MEG9 & maternally expressed 9 & 1.9449 & 0.0146 & Up \\
\hline LINC01410 & long intergenic non-protein coding RNA 1410 & 1.6411 & 0.0334 & Up \\
\hline CYP17A1 & cytochrome P450 family 17 subfamily A member 1 & 4.1951 & 0.0005 & Down \\
\hline OR10AB1P & olfactory receptor family 10 subfamily $\mathrm{AB}$ member 1 pseudogene & 4.0068 & 0.0146 & Down \\
\hline MYH14 & myosin heavy chain 14 & 3.7783 & 0.0317 & Down \\
\hline LRRTM3 & leucine rich repeat transmembrane neuronal 3 & 3.6646 & 0.0168 & Down \\
\hline GRIN3A & glutamate ionotropic receptor NMDA type subunit $3 \mathrm{~A}$ & -3.1104 & 0.0445 & Down \\
\hline HS3ST5 & heparan sulfate-glucosamine 3 -sulfotransferase 5 & -2.9968 & 0.0168 & Down \\
\hline NBAS & NBAS subunit of NRZ tethering complex & -2.8992 & 0.0146 & Down \\
\hline FNDC9 & fibronectin type III domain containing 9 & -2.8611 & 0.0419 & Down \\
\hline H1-4 & H1.4 linker histone, cluster member & -2.8427 & 0.0146 & Down \\
\hline CRYAB & crystallin alpha B & -2.7802 & 0.0146 & Down \\
\hline NXPH3 & neurexophilin 3 & -2.5502 & 0.0348 & Down \\
\hline MYL3 & myosin light chain 3 & -2.5310 & 0.0437 & Down \\
\hline CMYA5 & cardiomyopathy associated 5 & -2.4531 & 0.0311 & Down \\
\hline AMIGO2 & adhesion molecule with Ig like domain 2 & -2.2807 & 0.0499 & Down \\
\hline SIK1B & salt inducible kinase 1B (putative) & -2.2002 & 0.0446 & Down \\
\hline EDIL3 & EGF like repeats and discoidin domains 3 & -2.1682 & 0.0311 & Down \\
\hline UBC & ubiquitin $\mathrm{C}$ & -1.1926 & 0.0499 & Down \\
\hline lnc-FANCC-1 & & 2.6996 & 0.0437 & Up \\
\hline lnc-KLHL28-1 & & 2.5450 & 0.0311 & Up \\
\hline $\operatorname{lnc}-\mathrm{TBCCD} 1-4$ & & 2.1511 & 0.0051 & $\mathrm{Up}$ \\
\hline lnc-CDC27-8 & & 2.1123 & 0.0146 & Up \\
\hline lnc-SPG21-45 & & 2.0232 & 0.0166 & Up \\
\hline AC137695.1 & & 2.0062 & 0.0146 & $\mathrm{Up}$ \\
\hline Lnc-NSUN6-1 & & 1.5671 & 0.0499 & $\mathrm{Up}$ \\
\hline Lnc-ZNF814-1 & & 1.3445 & 0.0146 & Up \\
\hline Lnc-METRNL-8 & & -2.8955 & 0.0311 & Down \\
\hline Lnc-METRNL-1 & & -2.5951 & 0.0437 & Down \\
\hline
\end{tabular}

Shown are gene symbols, gene names, fold change $(\log 2 \mathrm{FC}), p$-adjusted value and gene expression status. Genes without names are non coding RNAs (LNCipedia GeneIDs) except AC137695.1. 
not present in the LS group and the following genes; SMIM28, HOXD10, PRSS12, NHLH2, MEG9, MAPK15, Lnc-ZNF814-1 and FNDC9, have no interactivity with any other DEG (Supplementary Text 1: SS-DEG-Net.tsv and $L S-D E G-N e t . t s v$ respectively store the 1018 and 650 interactions that can be viewed with Cytoscape).

Filtering the GSE49711 dataset with the query criteria of Table 3 yielded 43 SS and 19 LS samples, respectively. DEGs that do not have an associated $N C B I$ GeneID were not found in this dataset, particularly those that are identified as long non-coding RNAs (lncRNAs). Only 25 of the 40 DEGs have expression data in this dataset. Based on the results of feature selection with scikit-learn and several classification tests, the following 16 features were selected for the machine learning construction of the training and test sets; EVX2, NHLH2, PRSS12, POU6F2, HOXD10, MAPK15, RTL1, LGR5, CYP17A1, OR10AB1P, MYH14, LRRTM3, GRIN3A, HS3ST5, CRYAB and NXPH3. The evaluation of the Support Vector Machines (SVM) and Artificial Neural Networks (ANN) models using 5-fold cross-validation resulted in an accuracy of $78 \%$ and $87 \%$ for SVM and ANN, respectively. By testing the ML models on the GSE49711 test set, ANN again achieved better results with an accuracy of $82 \%$ of samples correctly classified as SS or LS compared to SVM which obtained an accuracy of 79\% (Table 2).

\section{DISCUSSION}

We aimed at identifying genes that are differentially expressed between high-risk SS and LS patients that could be potential prognostic indicators and or therapeutic targets. The results of the DGE analysis between the two groups showed the upregulation and downregulation of genes associated with neuroblastoma and other cancers.

\section{Differentially expressed genes}

\section{Upregulated genes}

The upregulated DEGs included some genes whose overexpression in the SS group have been correlated with poor survival in neuroblastoma and other cancers. Higher expression levels of NHLH2 were found to be higher in unfavourable neuroblastomas and was significantly associated with a poor prognosis [22]. Additional roles of NHLH2 in the obesity and fertility has also been uncovered $[23,24]$. The upregulation of PRSS12 in this study is similar to the results of Hiyama et al. [25], which reported the overexpression of PRSS12 in neuroblastoma tumors with high telomerase activity correlating with unfavourable tumors. Serine proteases are often altered and significantly upregulated in cancer as malignant cells need proteolytic activities to enable their growth, survival

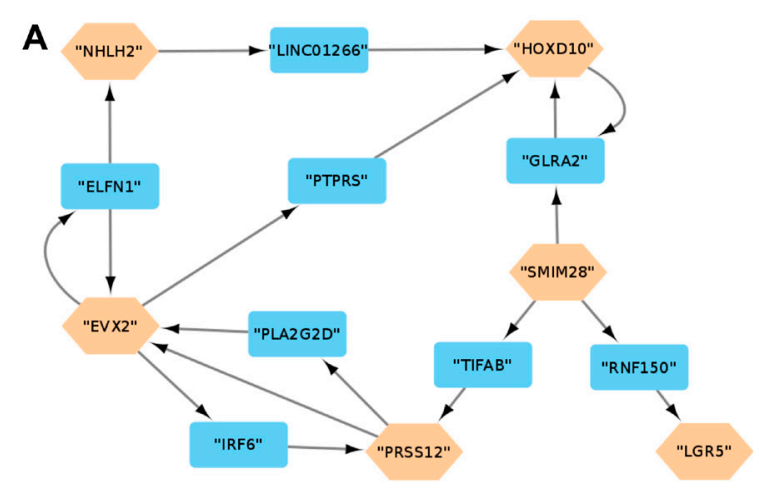

C
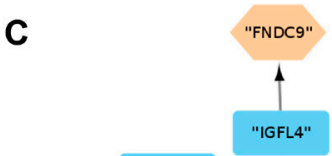
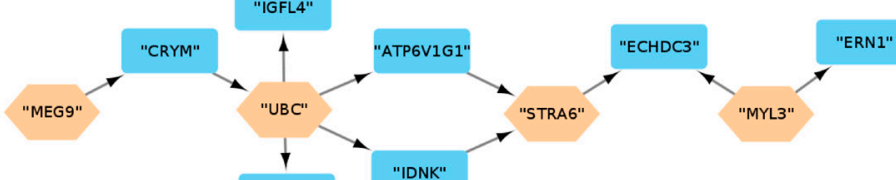

"IDNK"
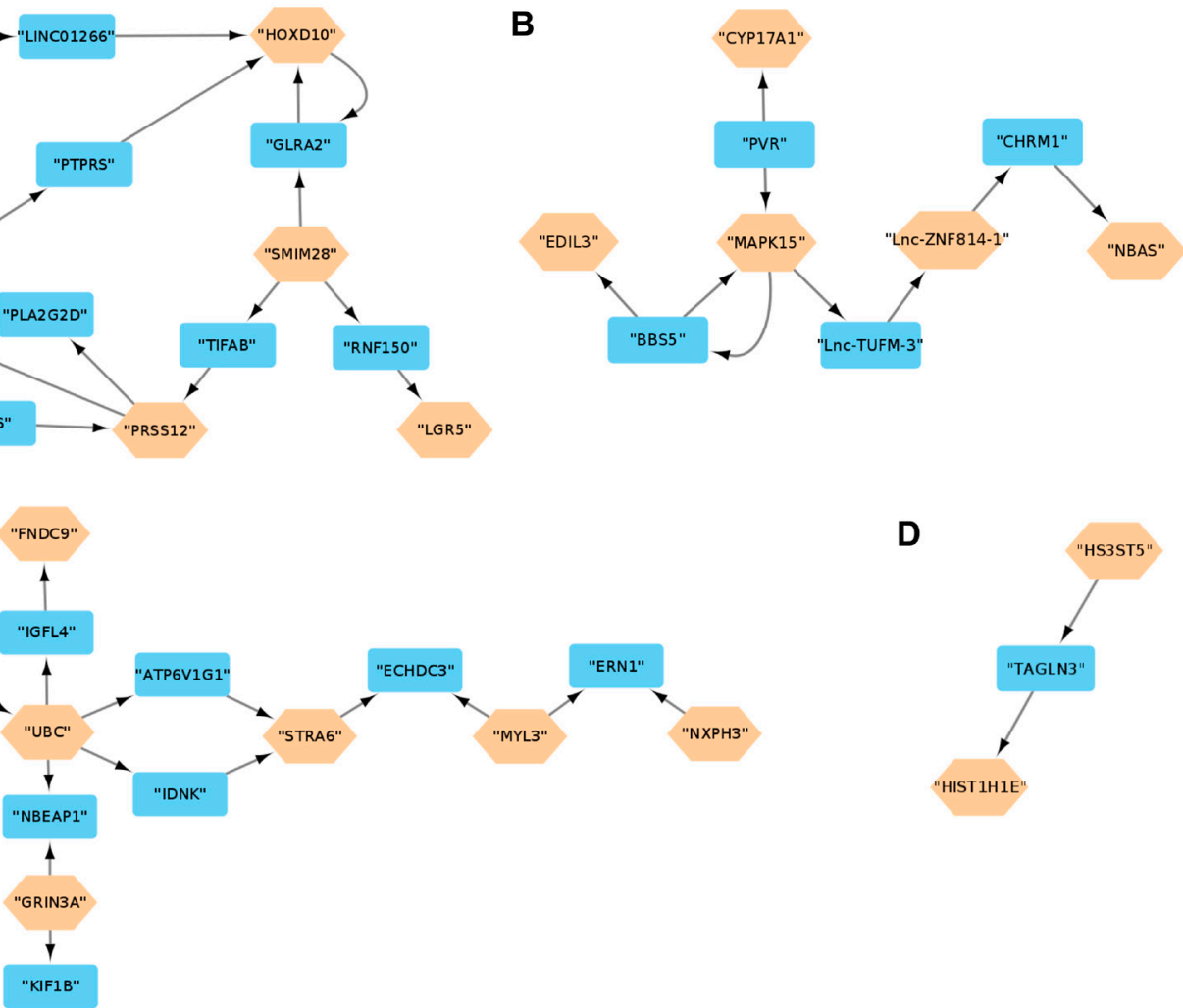

D

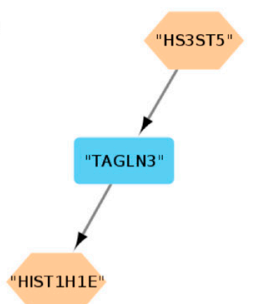

Figure 2: (A-D) Gene Regulatory Network Analysis. Regulatory networks for the DEG interactions in the SS group. The brown hexagonal nodes are DEGs and the blue rectangles are intermediate genes connecting two DEGs. DEGs that do not interact with other DEGs are omitted. 


\section{Table 2: Machine learning results for the classification of the GSE49711 test set of high-risk neuroblastoma samples with SVM and $A$ NN models}

\begin{tabular}{lccccc}
\hline & \multicolumn{3}{c}{ SVM } & & \multicolumn{2}{c}{ ANN } \\
\hline Class & Precision & & Recall & Precision & Recall \\
Short Survival & 0.87 & & 0.81 & 0.86 & 0.88 \\
Long Survival & 0.64 & & 0.74 & 0.72 & \\
Accuracy & & $79 \%$ & & & $82 \%$ \\
\hline
\end{tabular}

and expansion. Our result support that upregulation of PRSS12 is indicative of poor survival in neuroblastoma. HOXD10 is a transcription factor whose expression is altered in many cancers. Its high expression gives cancer cells proliferative and migratory abilities [26]. Elevated expression of HOXD genes including HOXD10 was reported to be associated with unfavourable prognosis and poor outcome in neuroblastoma [27], which supports our results indicating a more aggressive disease in the SS group. LGR5 is a stem cell marker which is highly expressed and associated with an aggressive phenotype in neuroblastoma $[28,29]$. It has also been associated with pancreatic ductal adenocarcinoma [30] and colorectal cancer [31]. LGR5 potentially contributes to stem cell maintenance and self renewal and is indicative of poor survival in high-risk neuroblastoma. SMIM28 is a less studied protein whose upregulation is indicative of poor survival in this study. Similar to our results, Jiang et al. [32] reported the upregulation of SMIM28 in prostate cancer. EVX2 is a homeobox transcription factor essential for vertebrate spinal cord neuronal specification [33]. POU6F2 belongs to the POU class homeobox family whose members are transcriptional regulators and is involved in hereditary predisposition to Wilms tumor, a pediatric malignancy of the kidney [34]. Functional studies are required to elucidate the roles of SMIM28, EVX2 and POU6F2 in high-risk neuroblastoma.

MAPK15, a protein kinase involved in many cellular activity including cell proliferation was upregulated in this study. Highest levels of MAPK15 was found in aggressive embryonal carcinomas and it acts by sustaining the progression of the cell cycle of embryonal carcinomas by limiting p 53 activation and preventing the facilitation of p53 dependent mechanisms that results to the arrest of the cell cycle [35]. Neuroblastoma is a malignancy of embryonal origin and upregulation of MAPK15 would be expected to facilitate tumor progression and indicative of aggressive disease and poor survival as in the SS group. RTL1, a paternally expressed imprinted gene highly expressed in the fetus, placenta and brain. It has been reported to be a driver of hepatocellular carcinoma [36]. Being a protease, it possibly promotes tumor invasion and metastasis in neuroblastoma tumors. Higher expression of RTL1 is thus suggestive of poor prognosis in neuroblastoma. Also upregulated is STRA6, a plasma membrane protein that transports retinol and is involved in a signalling mediated by JAK2, STAT3 and STAT5
[37]. Its upregulation indicates poor survival in our study possibly through its maintenance of cancer stem cells and promotion of tumor formation as reported in colorectal cancer $[37,38]$.

Ten IncRNAs were upregulated in this study. Three of these (lnc-SPG21-45, lnc-NSUN6-1 and lncKLHL28-1) are antisense to ANKDD1A, CACNB2 and $\mathrm{C} 14$ orf28 genes, respectively, which are associated with other cancers and diseases, possibly by regulating their expression. C14orf28 has been observed to be overexpressed in colorectal cancer cells, promoting proliferation, migration and invasion [39]. ANKDD1A has been described as a functional tumor suppressor with germline variants predicting poor patient outcomes in low grade glioma [40], and is frequently methylated in glioblastoma multiforme [41] and in clinically nonfunctioning pituitary adenomas [42]. CACNB2 is a calcium channel protein linked to diabetic retinopathy [43], bipolar disorder [44], hypertension [45] and autism spectrum disorders [46]. The role of calcium signalling in cancer has been reviewed by [47-49]. MEG9, is located in an imprinted non-coding RNA genomic region, DLK1DIO3 [50, 51]. LINC01410 is a lncRNA highly expressed in pancreatic cancer tissues and cell lines [52]. High expression in cholangiocarcinoma and gastric cancer patients have been associated with poor prognosis and survival $[53,54]$. These lncRNAs may be facilitating the promotion of tumor progression, proliferation and invasion which might have impacted the survival of patients in the short survival group. It is well established that metastasis is the primary cause of cancer mortality. Functional studies are required to evaluate the roles of these lncRNAs in neuroblastoma.

\section{Downregulated genes}

The downregulated genes include genes such as AMIGO2, LRRTM3, GRIN3A, MYH14, EDIL3, FNDC9, involved in neural function and development, and in extracellular matrix (ECM) organization. AMIGO2 is a transmembrane molecule expressed in neuronal tissues and participates in their formation [55]. EDIL3 is an inducer of the epithelial-mesenchymal transition, that promotes angiogenesis and invasion in hepatocellular carcinoma [56]. FNDC9 which exhibits biased expressed in the brain is an ECM protein involved in tumorigenesis in different cancers [57]. MYH14 is a myosin, an actin-dependent motor protein that plays a role in neuritogenesis. Members 
Table 3: Query criteria for short survival (SS) and long survival (LS) sample selection

\begin{tabular}{lll}
\hline Field & SS values & LS values \\
\hline Diagnostic category & Neuroblastoma & Neuroblastoma \\
INSS stage & Stage 4 & Stage 4 \\
COG risk group & High risk & High risk \\
Vital status & Dead & Alive \\
Overall survival time & 730 days & 2555 days \\
\hline
\end{tabular}

of the myosin superfamily have been known to enhance or suppress tumor progression [58]. MYH14 could be suppressing tumor progression in high-risk neuroblastoma. The downregulation of these ECM associated genes could be promoting the invasion and metastasis of neuroblastoma tumors.

OR10AB1P belongs to the olfactory receptor family of genes. Olfactory receptors are expressed in various human tissues and are involved in different cellular processes such as migration and proliferation. Some are biomarkers for prostate, lung and small intestine carcinoma tissues [59]. Decreased expression of CRYAB indicated its tumor suppressor function in bladder cancer [60]. It may thus also be functioning as a tumor suppressor in neuroblastoma. Ubiquitin $\mathrm{C}(\mathrm{UBC})$ is a polyubiquitin precursor. Ubiquitination has been associated with many cellular processes which play roles in tumorigenesis.

CYP17A1 is a key enzyme in the steroidogenic pathway with restricted expression in the adrenal gland. Neuroblastoma tumors commonly occur in the adrenal medulla. The downregulation of CYP17A1 may be an indicator of poor prognosis in neuroblastoma. LRRTM3 has high expression in the brain and belongs to a group of proteins involved in nervous system development. How it contributes to neuroblastoma remains to be investigated but it is currently a candidate gene for alzheimer's disease $[61,62]$, a neurological disorder. NBAS is thought to be involved in golgi-to-ER transport and is typically amplified in MYCN-amplified neuroblastoma tumors [63]. GRIN3A is a glutamate receptor that promotes nerve outgrowth [64]. The downregulation of GRIN3A may suggest a higher level of disease because glutamate is a major excitatory neurotransmitter in the CNS that is involved in many neuronal processes. Functional studies are required to investigate how these genes contribute to poor survival in neuroblastoma.

\section{Gene and disease ontology enrichment analyses}

The molecular function activities; MAP kinase activity, retinol binding and RNA polymerase II activating transcription factor binding, enriched by the upregulated genes MAPK15, STRA16 and NHLH2, respectively, are activities that promote tumor cell proliferation. Deregulation of the MAPK signalling was associated with cancer development, progression and cell proliferation $[65,66]$. Retinol binding through the STRA6 upregulation activates a signalling cascade that is found to play a role in cancer initiation, maintenance and growth [38]. Furthermore, the increased global transcription activity (activation of RNA polymerase II) indicates an intensity of a rapid proliferation of cancer cells [67]. These activities again demonstrate the aggressiveness of the neuroblastoma tumors in SS patients compared to LS patients.

The enriched diseases by the upregulated and downregulated genes, particularly the disorders inducing heart failure (Cardiomyopathy and Congenital heart disease) and respiratory illness (Diaphragmatic dysfunction) may have negative impact on survival $[68,69]$, and could be the cause of death in the SS neuroblastoma patients. There is no indication in the clinical information accompanying the gene expression count dataset if the patients suffered from additional disorders in addition to neuroblastoma. However, it is reported that high-risk neuroblastoma survivors treated with intensive multimodality therapy are at risk for a broad variety of treatment-related late effects including cardiac dysfunction, bone development disease, pulmonary dysfunction and impaired fertility [70]. These treatmentrelated morbidities were enriched by the upregulated and downregulated DEGs (Figure 1B and 1C), suggesting that the cause of death in patients with SS neuroblastoma may be due to treatment complications.

\section{Regulatory network analysis}

After the application of a weight threshold of 0.00251 to the GENIE3 output, the numbers of interactions involving the DEGs in the SS and LS groups were 1018 and 650, respectively. There is a clear substantial difference in number of interactions between the two groups, indicating a higher cellular/tumor activity in the SS group which could be a sign of tumor aggressiveness in patients with SS neuroblastoma. In addition, it is noticeable from the networks in Figure 2 the importance of the genes SMIM28, MAPK15 and UBC as origins of most of the interactions. The role of MAPK15 and $\mathrm{UBC}$ in tumorigenesis has been reported in many previously discussed scientific works, while SMIM28 is a less studied gene with an unclear role in cancer. However, observation of the role of the final target genes of SMIM28 in Figure 2A network, which are LGR5 and HOXD10, can shed light on the role of this gene. As reported previously, both LGR5 and HOXD10 were associated with cancer cell proliferation and tumor aggressiveness in neuroblastoma. Thus, it is 
possible that SMIM28 (Small Integral Membrane Protein 28 ) is part of a signalling pathway whose role is to accelerate neuroblastoma tumor proliferation, making it a possible new gene target therapy in high-risk neuroblastoma cancer. Further investigations are required to elucidate precisely the role of SMIM28 in the aggressiveness of neuroblastoma tumors.

\section{Machine learning}

Although not all the DEGs were included in the training and testing of the machine learning models, the obtained prediction results were significantly good. The ANN model obtained the highest accuracy of $82 \%$ for the classification of the external neuroblastoma samples (GSE49711) into short and long survival classes. This high classification accuracy makes it possible to consider that the DEG expression profiles have been preserved in the various high-risk neuroblastoma tumors, although neuroblastoma is known to be heterogeneous. Therefore, the DEG list can serve as prognostic indicators (genetic signature) for survival time in high-risk neuroblastoma patients and can be targets for drug discovery analyses. Relatively similar to our study, other studies have proposed genetic signatures for prognostic stratification of patients with neuroblastoma. Liu et al. [71] used an unsupervised biclustering machine learning technique to find high-risk neuroblastoma subtypes. They proposed a signature of 238 neuroblastoma-specific immune genes to identify ultra high-risk and high-risk neuroblastoma subtypes. Russo et al. [72] applied K-means clustering to 27 kinome gene signature to identify ultra high-risk subtypes of high-risk neuroblastoma. Formicola et al. [73] used Cox regression and Kaplan-Meier analysis methods to propose a 18-gene expression based risk scoring system to predict overall survival of patients with stage 4 neuroblastoma. We demonstrated the use of a shorter signature in a 16-gene expression classifier based on survival time to stratify high-risk neuroblastomas into SS (ultra high-risk) and LS subtypes. The number of genes in our classifier should provide the advantage of being less costly and easier to implement.

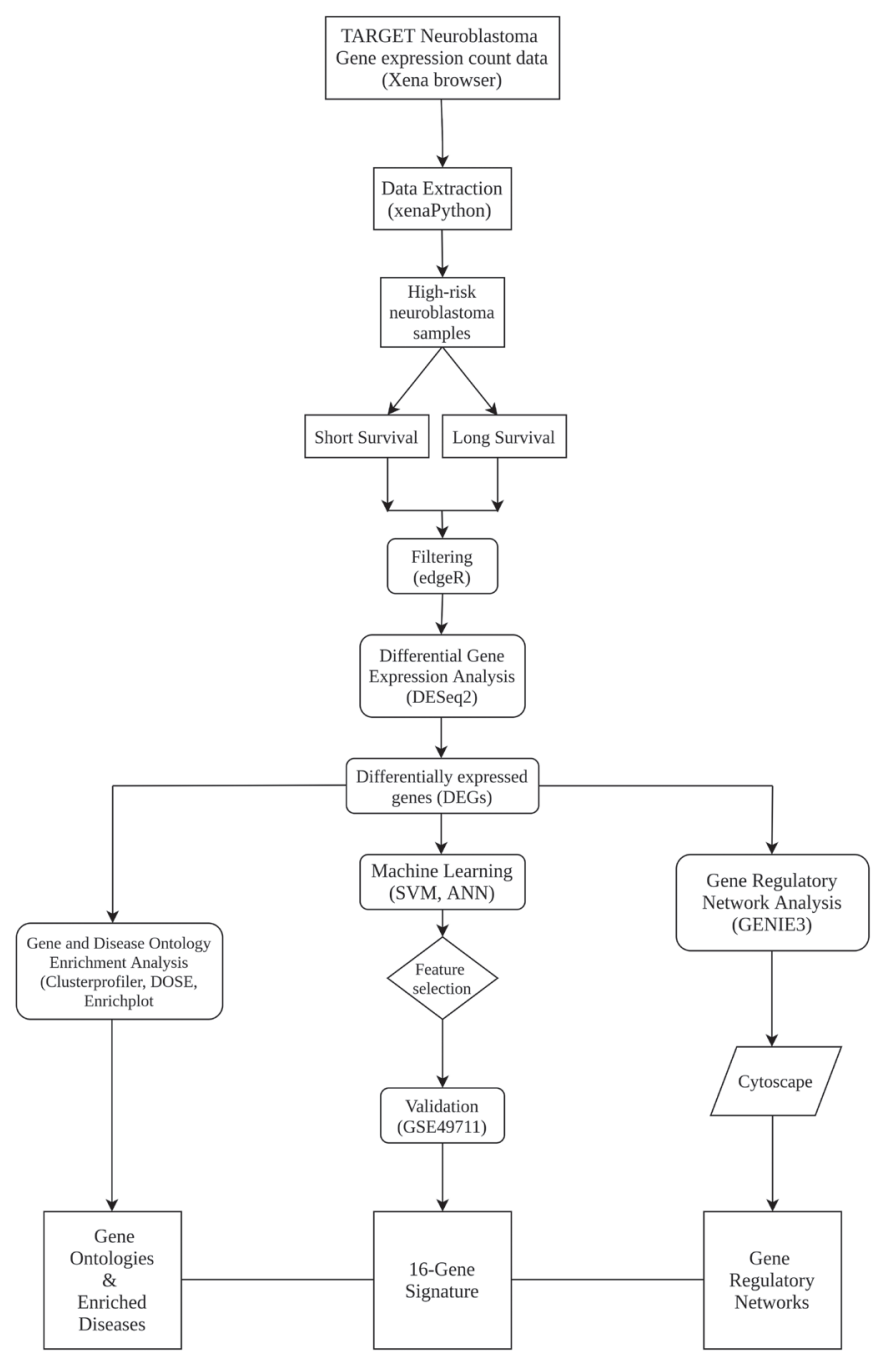

Figure 3: Workflow outlining the steps and methods undertaken in this study. 


\section{MATERIALS AND METHODS}

The workflow describing the steps and methods undertaken in this study is illustrated in Figure 3. It includes 5 essential steps; dataset retrieval, differential gene expression, disease/gene ontology enrichment, gene regulatory network inference and machine learning.

\section{Datasets}

The Therapeutically Applicable Research to Generate Effective Treatments (TARGET) initiative employed comprehensive molecular characterization of hard-to-treat childhood cancers which included neuroblastoma. TARGET data is accessible via the TARGET data matrix as well as via the Xena browser. The Xena browser is a web based visualization and exploration tool for multi-omic data large public repositories and private datasets [74]. The TARGET neuroblastoma dataset in the Xena database $[74,75]$ is composed of high-risk neuroblastoma samples with available clinical information. Gene expression RNA-seq read counts of the TARGET neuroblastoma dataset (dataset ID: TARGETNBL. htseq_counts. tsv) were obtained from the GDC hub in Xena browser using xenaPython package. Fields used in querying the dataset are described in Table 3 . The dataset itself was composed of 151 samples in total. Querying the dataset with the above fields returned 32 neuroblastoma samples; 20 of which with an overall survival time of less than 730 days ( 2 years) and vital status is "dead" were considered short survival (SS), while 12 samples with an overall survival time greater than 2555 days ( 7 years) and vital status is "alive" were considered long survival (LS).

\section{Differential gene expression analysis}

Normalized expression counts were converted to raw counts and filtered to remove low expressed genes using the edgeR filterByExpr function in R [76]. We then performed a differential gene expression (DGE) analysis between the short and long survival groups using the DESeq2 package in R [77]. DESeq2 uses shrinkage estimators for dispersion and fold change for its comparative differential gene expression estimation [77]. Differentially expressed genes (DEGs) were selected by meeting criteria of adjusted $p$-value $<0.05$. Gene Ontology (GO) enrichment analysis was carried out to functionally annotate the DEGs using clusterProfiler [78] and visualized using enrichplot. The DOSE R library [79] was used to detect the diseases enriched by the upregulated and downregulated DEGs.

\section{Machine learning}

Scikit-learn [80] and LibSVM [81] libraries were used for machine learning (ML) model creation and classification tasks. Features (genes) not present in the test dataset (GSE49711) were not used for machine learning prediction. The top upregulated and downregulated genes used as features for machine learning (ML) prediction of patient survival were; EVX2, NHLH2, PRSS12, POU6F2, HOXD10, MAPK15, RTL1, LGR5, CYP17A1, OR10AB1P, MYH14, LRRTM3, GRIN3A, HS3ST5, CRYAB, NXPH3. The features were extracted from the log-normalized counts data. For LibSVM, the feature values of the training and test sets were scaled using a built-in python script. With regards to ANN, the feature values of the training and test sets were scaled with the Scikit-learn MinMaxScaler function. Algorithms used for training and evaluation of the models include; Support Vector Machines (SVM) and Artificial Neural Networks (ANN). 5-fold cross validation was done to determine the best parameters of the SVM and ANN models which was then applied to our test samples. Both models, created with LibSVM and ANN were applied to predict the classification of samples in an external dataset (GSE49711) with same sample characteristics (overall survival $<730$ days with vital status as dead for SS samples, and overall survival $>$ 2555 days with vital status as alive for LS samples). The evaluation metrics for the LibSVM and ANN models were precision, recall and accuracy.

\section{Regulatory networks}

The GENIE3 package [82] in R was used for genetic regulatory network inference analysis. The GENIE3 algorithm uses a Random Forest or Extra Randomized Trees approach to infer gene regulatory networks from gene expression data [82]. It outputs a ranked list of each pairwise comparison from the most to the least confident regulatory connection. The library was run on the gene expression counts from the SS and LS samples separately. For the output analysis, only connections involving the DEGs were considered. In addition, a weighting threshold of 0.00251 was applied to reduce the large number of connections and also to focus on high confident regulatory connections. Cytoscape [83] was used for the visualization of gene regulatory networks.

\section{CONCLUSIONS}

The DGE analysis is a powerful technique for identifying DEGs in a studied condition. In this study, using DESeq 2 we identified 40 DEGs between SS and LS neuroblastoma samples. Many of the DEGs were found to be related to different cancers (including neuroblastoma), thus strengthening their possibility of being associated with neuroblastoma. The ML models based on 16 DEGs were capable of stratifying high-risk neuroblastoma samples on the basis of survival time, demonstrating their ability to be used as a genetic signature or prognostic indicators of survival in high-risk neuroblastoma 
patients. This study furthers our understanding of the molecular mechanisms of neuroblastoma in high-risk patients. We identified SMIM28 gene to be critical for tumor proliferation making it as a possible gene therapy target. Nevertheless, additional studies are required to elucidate the role of SMIM28 in the pathogenesis of neuroblastoma. Finally, prognostic stratification of highrisk neuroblastoma patients will help clinicians in making better therapeutic and patient management decisions.

\section{Abbreviations}

DGE: Differential Gene Expression; DEG: Differentially Expressed Gene; GO: Gene Ontology; DO: Disease Ontology; ML: Machine Learning; ANN: Artificial Neural Networks; SVM: Support Vector Machines; TARGET: Therapeutically Applicable Research to Generate Effective Treatments.

\section{Author contributions}

$\mathrm{AC}$ and $\mathrm{HB}$ funded the project. $\mathrm{JG}, \mathrm{AG}$ and $\mathrm{HB}$ designed the study. AG and HB implemented the analysis. $\mathrm{AG}, \mathrm{AF}$ and HB wrote the manuscript. All authors read and approved the final manuscript.

\section{ACKNOWLEDGMENTS}

We want to acknowledge the support received from the South African Medical Research Council and National Research Foundation of South Africa.

\section{CONFLICTS OF INTEREST}

Authors have no conflicts of interest to declare.

\section{FUNDING}

The work reported herein was made possible through funding by the South African Medical Research Council through its Division of Research Capacity Development under the Mid-Career Scientist Programme from funding received from the South African National Treasury. This work was also supported by the South African Research Chairs Initiative of the Department of Science and Technology and National Research Foundation of South Africa (Grant ID 64751). The content hereof is the sole responsibility of the authors and does not necessarily represent the official views of the SAMRC.

\section{REFERENCES}

1. Maris JM. Recent Advances in Neuroblastoma. N Engl J Med. 2010; 362:2202-2211. https://doi.org/10.1056/ NEJMra0804577. [PubMed]
2. Smith MA, Seibel NL, Altekruse SF, Ries LA, Melbert DL, O'Leary M, Smith FO, Reaman GH. Outcomes for children and adolescents with cancer: challenges for the twenty-first century. J Clin Oncol. 2010; 28:2625-2634. https://doi. org/10.1200/JCO.2009.27.0421. [PubMed]

3. Ward E, DeSantis C, Robbins A, Kohler B, Jemal A. Childhood and adolescent cancer statistics, 2014. CA Cancer J Clin. 2014; 64:83-103. https://doi.org/10.3322/ caac.21219. [PubMed]

4. Zhang L, Lv C, Jin Y, Cheng G, Fu Y, Yuan D, Tao Y, Guo Y, Ni X, Shi T. Deep learning-based multi-omics data integration reveals two prognostic subtypes in highrisk neuroblastoma. Front Genet. 2018; 9:477. https://doi. org/10.3389/fgene.2018.00477. [PubMed]

5. Johnsen JI, Dyberg C, Wickström M. Neuroblastoma-A Neural Crest Derived Embryonal Malignancy. Front Mol Neurosci. 2019; 12:9. https://doi.org/10.3389/ fnmol.2019.00009. [PubMed]

6. Baali I, Acar DAE, Aderinwale TW, HafezQorani S, Kazan H. Predicting clinical outcomes in neuroblastoma with genomic data integration. Biol Direct. 2018; 13:20. https:// doi.org/10.1186/s13062-018-0223-8. [PubMed]

7. Oeffinger KC, Mertens AC, Sklar CA, Kawashima T, Hudson MM, Meadows AT, Friedman DL, Marina N, Hobbie W, Kadan-Lottick NS, Schwartz CL, Leisenring W, Robison LL, et al. Chronic health conditions in adult survivors of childhood cancer. N Engl J Med. 2006; 355:1572-1582. https://doi.org/10.1056/NEJMsa060185. [PubMed]

8. Yu AL, Gilman AL, Ozkaynak MF, London WB, Kreissman SG, Chen HX, Smith M, Anderson B, Villablanca JG, Matthay KK, Shimada H, Grupp SA, Seeger R, et al. AntiGD2 antibody with GM-CSF, interleukin-2, and isotretinoin for neuroblastoma. N Engl J Med. 2010; 363:1324-1334. https://doi.org/10.1056/NEJMoa0911123. [PubMed]

9. Mossé YP, Laudenslager M, Longo L, Cole KA, Wood A, Attiyeh EF, Laquaglia MJ, Sennett R, Lynch JE, Perri P, Laureys G, Speleman F, Kim C, et al. Identification of ALK as a major familial neuroblastoma predisposition gene. Nature. 2008; 455:930-935. https://doi.org/10.1038/ nature 07261. [PubMed]

10. Valentijn LJ, Koster J, Zwijnenburg DA, Hasselt NE, van Sluis P, Volckmann R, van Noesel MM, George RE, Tytgat GA, Molenaar JJ, Versteeg R. TERT rearrangements are frequent in neuroblastoma and identify aggressive tumors. Nat Genet. 2015; 47:1411-1414. https://doi.org/10.1038/ ng. 3438. [PubMed]

11. Cheung NK, Zhang J, Lu C, Parker M, Bahrami A, Tickoo SK, Heguy A, Pappo AS, Federico S, Dalton J, Cheung IY, Ding L, Fulton B, et al. Association of Age at Diagnosis and Genetic Mutations in Patients with Neuroblastoma. JAMA. 2012; 307:1062-1071. https://doi.org/10.1001/ jama.2012.228. [PubMed]

12. Peifer M, Hertwig F, Roels F, Dreidax D, Gartlgruber M, Menon R, Krämer A, Roncaioli JL, Sand F, Heuckmann JM, Ikram F, Schmidt R, Ackermann S, et al. Telomerase 
activation by genomic rearrangements in high-risk neuroblastoma. Nature. 2015; 526:700-704. https://doi. org/10.1038/nature14980. [ [PubMed]

13. Maris JM, Mosse YP, Bradfield JP, Hou C, Monni S, Scott RH, Asgharzadeh S, Attiyeh EF, Diskin SJ, Laudenslager M, Winter C, Cole KA, Glessner JT, et al. Chromosome 6 p22 locus associated with clinically aggressive neuroblastoma. N Engl J Med. 2008; 358:2585-2593. https://doi.org/10.1056/NEJMoa0708698. [PubMed]

14. Cimmino F, Avitabile M, Diskin SJ, Vaksman Z, Pignataro P, Formicola D, Cardinale A, Testori A, Koster J, de Torres C, Devoto M, Maris JM, Iolascon A, et al. Fine mapping of 2q35 high-risk neuroblastoma locus reveals independent functional risk variants and suggests full-length BARD1 as tumor-suppressor. Int J Cancer. 2018; 143:2828-2837. https://doi.org/10.1002/ijc.31822. [PubMed]

15. Capasso M, Diskin S, Cimmino F, Acierno G, Totaro F, Petrosino G, Pezone L, Diamond M, McDaniel L, Hakonarson H, Iolascon A, Devoto M, Maris JM. Common genetic variants in NEFL influence gene expression and neuroblastoma risk. Cancer Res. 2014; 74:6913-6924. https://doi.org/10.1158/0008-5472.CAN-14-0431. [PubMed]

16. Diskin SJ, Hou C, Glessner JT, Attiyeh EF, Laudenslager M, Bosse K, Cole K, Mosse YP, Wood A, Lynch JE, Pecor K, Diamond $\mathrm{M}$, Winter C, et al. Copy number variation at 1q21.1 associated with neuroblastoma. Nature. 2009; 459:987-991. https://doi.org/10.1038/nature08035. [PubMed]

17. Wang K, Diskin SJ, Zhang H, Attiyeh EF, Winter C, Hou C, Schnepp RW, Diamond M, Bosse K, Mayes PA, Glessner J, Kim C, Frackelton E, et al. Integrative genomics identifies LMO1 as a neuroblastoma oncogene. Nature. 2011; 469:216-220. https://doi.org/10.1038/nature09609. [PubMed]

18. Bosse KR, Diskin SJ, Cole KA, Wood AC, Schnepp RW, Norris G, Nguyen LB, Jagannathan J, Laquaglia M, Winter C, Diamond M, Hou C, Attiyeh EF, et al. Common variation at BARD1 results in the expression of an oncogenic isoform that influences neuroblastoma susceptibility and oncogenicity. Cancer Res. 2012; 72:2068-2078. https://doi. org/10.1158/0008-5472.CAN-11-3703. [PubMed]

19. Oldridge DA, Wood AC, Weichert-Leahey N, Crimmins I, Sussman R, Winter C, McDaniel LD, Diamond M, Hart LS, Zhu S, Durbin AD, Abraham BJ, Anders L, et al. Genetic predisposition to neuroblastoma mediated by a LMO1 super-enhancer polymorphism. Nature. 2015; 528:418-421. https://doi.org/10.1038/nature15540. [ubMed]

20. Russell MR, Penikis A, Oldridge DA, Alvarez-Dominguez JR, McDaniel L, Diamond M, Padovan O, Raman P, Li Y, Wei JS, Zhang S, Gnanchandran J, Seeger R, et al. CASC15-S is a tumor suppressor lncRNA at the $6 \mathrm{p} 22$ neuroblastoma susceptibility locus. Cancer Res. 2015; 75:3155-3166. https://doi.org/10.1158/0008-5472.CAN14-3613. [PubMed]
21. Tonini GP, Capasso M. Genetic predisposition and chromosome instability in neuroblastoma. Cancer Metastasis Rev. 2020; 39:275-285. https://doi.org/10.1007/ s10555-020-09843-4. [PubMed]

22. Aoyama M, Ozaki T, Inuzuka H, Tomotsune D, Hirato J, Okamoto Y, Tokita H, Ohira M, Nakagawara A. LMO3 interacts with neuronal transcription factor, HEN2, and acts as an oncogene in neuroblastoma. Cancer Res. 2005; 65:4587-4597. https://doi.org/10.1158/0008-5472.CAN-044630. [PubMed]

23. Good DJ, Braun T. NHLH2: At the intersection of obesity and fertility. Trends Endocrinol Metab. 2013; 24:385-390. https://doi.org/10.1016/j.tem.2013.04.003. [PubMed]

24. Vella KR, Burnside AS, Brennan KM, Good DJ. Expression of the hypothalamic transcription factor nhlh2 is dependent on energy availability. J Neuroendocrinol. 2007; 19:499510. https://doi.org/10.1111/j.1365-2826.2007.01556.x. [PubMed]

25. Hiyama E, Hiyama K, Nishiyama M, Reynolds CP, Shay JW, Yokoyama T. Differential gene expression profiles between neuroblastomas with high telomerase activity and low telomerase activity. J Pediatr Surg. 2003; 38:1730 1734. $\quad$ https://doi.org/10.1016/j.jpedsurg.2003.08.042. [PubMed]

26. Hakami F, Darda L, Stafford P, Woll P, Lambert DW, Hunter KD. The roles of HOXD10 in the development and progression of head and neck squamous cell carcinoma (HNSCC). Br J Cancer. 2014; 111:807-816. https://doi. org/10.1038/bjc.2014.372. [PubMed]

27. Kocak H, Ackermann S, Hero B, Kahlert Y, Oberthuer A, Juraeva D, Roels F, Theissen J, Westermann F, Deubzer H, Ehemann V, Brors B, Odenthal M, et al. Hox-C9 activates the intrinsic pathway of apoptosis and is associated with spontaneous regression in neuroblastoma. Cell Death Dis. 2013; 4:e586. https://doi.org/10.1038/cddis.2013.84. [PubMed]

28. Vieira GC, Chockalingam S, Melegh Z, Greenhough A, Malik S, Szemes M, Park JH, Kaidi A, Zhou L, Catchpoole D, Morgan R, Bates DO, Gabb PJ, et al. LGR5 regulates pro-survival MEK/ERK and proliferative Wnt/ $\beta$-catenin signalling in neuroblastoma. Oncotarget. 2015; 6:4005340067. https://doi.org/10.18632/oncotarget.5548. [PubMed]

29. Forgham H, Johnson D, Carter N, Veuger S, Carr-Wilkinson J. Stem Cell Markers in Neuroblastoma-An Emerging Role for LGR5. Front Cell Dev Biol. 2015; 3:77. https:// doi.org/10.3389/fcell.2015.00077. [PubMed]

30. Kuraishi Y, Uehara T, Kobayashi Y, Nakajima T, Watanabe T, Shimizu A, Ota H, Tanaka E. Correlation of clinicopathological features and leucine-rich repeatcontaining G-protein-coupled receptor 5 expression in pancreatic ductal adenocarcinoma. Pathol Res Pract. 2019; 215:152623. https://doi.org/10.1016/j.prp.2019.152623. [PubMed] 
31. Morgan R, Mortensson E, Williams A. Targeting LGR5 in colorectal cancer: therapeutic gold or too plastic? Br J Cancer. 2018; 118:1410-1418. https://doi.org/10.1038/ s41416-018-0118-6. [PubMed]

32. Jiang T, Guo J, Hu Z, Zhao M, Gu Z, Miao S. Identification of potential prostate cancer-related pseudogenes based on competitive endogenous RNA network hypothesis. Med Sci Monit. 2018; 24:4213-4239. https://doi.org/10.12659/ MSM.910886. [PubMed]

33. Juárez-Morales JL, Schulte CJ, Pezoa SA, Vallejo GK, Hilinski WC, England SJ, de Jager S, Lewis KE. Evx1 and Evx2 specify excitatory neurotransmitter fates and suppress inhibitory fates through a Pax2-independent mechanism. Neural Dev. 2016; 11:5. https://doi.org/10.1186/s13064016-0059-9. [PubMed]

34. Perotti D, De Vecchi G, Testi MA, Lualdi E, Modena P, Mondini P, Ravagnani F, Collini P, Di Renzo F, Spreafico F, Terenziani M, Sozzi G, Fossati-Bellani F, et al. Germline mutations of the POU6F2 gene in Wilms tumors with loss of heterozygosity on chromosome 7p14. Hum Mutat. 2004; 24:400-407. https://doi.org/10.1002/humu.20096. [PubMed]

35. Rossi M, Colecchia D, Ilardi G, Acunzo M, Nigita G, Sasdelli F, Celetti A, Strambi A, Staibano S, Croce CM, Chiariello M. MAPK15 upregulation promotes cell proliferation and prevents DNA damage in male germ cell tumors. Oncotarget. 2016; 7:20981-20998. https://doi. org/10.18632/oncotarget.8044. [PubMed]

36. Riordan JD, Keng VW, Tschida BR, Scheetz TE, Bell JB, Podetz-Pedersen KM, Moser CD, Copeland NG, Jenkins NA, Roberts LR, Largaespada DA, Dupuy AJ. Identification of rtl1, a retrotransposon-derived imprinted gene, as a novel driver of hepatocarcinogenesis. PLoS Genet. 2013; 9:e1003441. https:// doi.org/10.1371/journal.pgen.1003441. [PubMed]

37. Berry DC, Levi L, Noy N. Holo-retinol-binding protein and its receptor STRA6 drive oncogenic transformation. Cancer Res. 2014; 74:6341-6351. https://doi.org/10.1158/00085472.CAN-14-1052. [PubMed]

38. Karunanithi S, Levi L, DeVecchio J, Karagkounis G, Reizes O, Lathia JD, Kalady MF, Noy N. RBP4-STRA6 pathway drives cancer stem cell maintenance and mediates high-fat diet-induced colon carcinogenesis. Stem Cell Reports. 2017; 9:438-450. https://doi.org/10.1016/j.stemcr.2017.06.002. [PubMed]

39. Yang X, Hu Y, Liu Y, Liu W, Zhao X, Liu M, Tang H. C14orf28 downregulated by miR-519d contributes to oncogenicity and regulates apoptosis and EMT in colorectal cancer. Mol Cell Biochem. 2017; 434:197-208. https://doi. org/10.1007/s11010-017-3049-2. [PubMed]

40. Chatrath A, Kiran M, Kumar P, Ratan A, Dutta A. The germline variants rs61757955 and rs34988193 are predictive of survival in lower grade glioma patients. Mol Cancer Res. 2019; 17:1075-1086. https://doi. org/10.1158/1541-7786.MCR-18-0996. [PubMed]

41. Feng J, Zhang Y, She X, Sun Y, Fan L, Ren X, Fu H, Liu C, Li P, Zhao C, Liu Q, Liu Q, Li G, et al. Hypermethylated gene ANKDD1A is a candidate tumor suppressor that interacts with FIH1 and decreases HIF $1 \alpha$ stability to inhibit cell autophagy in the glioblastoma multiforme hypoxia microenvironment. Oncogene. 2019; 38:103-119. https:// doi.org/10.1038/s41388-018-0423-9. [PubMed]

42. Cheng S, Xie W, Miao Y, Guo J, Wang J, Li C, Zhang Y. Identification of key genes in invasive clinically nonfunctioning pituitary adenoma by integrating analysis of DNA methylation and mRNA expression profiles. J Transl Med. 2019; 17:407. https://doi.org/10.1186/s12967-01902148-3. [PubMed]

43. Vuori N, Sandholm N, Kumar A, Hietala K, Syreeni A, Forsblom C, Juuti-Uusitalo K, Skottman H, Imamura M, Maeda S, Summanen PA, Lehto M, Groop PH, et al. CACNB2 Is a novel susceptibility gene for diabetic retinopathy in type 1 diabetes. Diabetes. 2019; 68:21652174. https://doi.org/10.2337/db19-0130. [PubMed]

44. Liu F, Gong X, Yao X, Cui L, Yin Z, Li C, Tang Y, Wang F. Variation in the CACNB2 gene is associated with functional connectivity of the Hippocampus in bipolar disorder. BMC Psychiatry. 2019; 19:62. https://doi.org/10.1186/s12888019-2040-8. [PubMed]

45. Niu Y, Gong Y, Langaee TY, Davis HM, Elewa H, Beitelshees AL, Moss JI, Cooper-DeHoff RM, Pepine CJ, Johnson JA. Genetic variation in the beta 2 subunit of the voltage-gated calcium channel (CACNB2) and pharmacogenetic association with adverse cardiovascular outcomes in the INternational VErapamil SR-Trandolapril STudy-GENEtic Substudy (INVEST-GENES). Circ Cardiovasc Genet. 2010; 3:548-555. https://doi. org/10.1161/CIRCGENETICS.110.957654. [PubMed]

46. Breitenkamp AFS, Matthes J, Nass RD, Sinzig J, Lehmkuhl G, Nürnberg P, Herzig S. Rare mutations of CACNB2 found in autism spectrum disease-affected families alter calcium channel function. PLoS One. 2014; 9:e95579. https://doi. org/10.1371/journal.pone.0095579. [PubMed]

47. Yang H, Zhang Q, He J, Lu W. Regulation of calcium signaling in lung cancer. J Thorac Dis. 2010; 2:52-56. [PubMed]

48. Stewart TA, Yapa KTDS, Monteith GR. Altered calcium signaling in cancer cells. Biochim Biophys Acta. 2015; 1848:2502-2511. https://doi.org/10.1016/j. bbamem.2014.08.016. [PubMed]

49. Cui C, Merritt R, Fu L, Pan Z. Targeting calcium signaling in cancer therapy. Acta Pharm Sin B. 2017; 7:3-17. https:// doi.org/10.1016/j.apsb.2016.11.001. [PubMed]

50. Hagan JP, O’Neill BL, Stewart CL, Kozlov SV, Croce CM. At least ten genes define the imprinted Dlk1-Dio3 cluster on Mouse chromosome 12qF1. PLoS One. 2009; 4:e4352. https://doi.org/10.1371/journal.pone.0004352. [PubMed]

51. Benetatos L, Hatzimichael E, Londin E, Vartholomatos G, Loher P, Rigoutsos I, Briasoulis E. The microRNAs within the DLK1-DIO3 genomic region: involvement in disease pathogenesis. Cell Mol Life Sci. 2013; 70:795-814. https:// doi.org/10.1007/s00018-012-1080-8. [ [PubMed] 
52. Cai M, Xu L, Shen L, Zhang J. [The expression of long non-coding RNA-LINC01410 in pancreatic cancer and its effect on proliferation and migration of pancreatic cancer cells]. [Article in Chinese]. Zhonghua Yi Xue Za Zhi. 2019; 99:1406-1411. https://doi.org/10.3760/cma.j.is sn.0376-2491.2019.18.010. [PubMed]

53. Zhang JX, Chen ZH, Chen DL, Tian XP, Wang CY, Zhou ZW, Gao Y, Xu Y, Chen C, Zheng ZS, Weng HW, Ye S, Kuang M, et al. LINC01410-miR-532-NCF2-NF-kB feedback loop promotes gastric cancer angiogenesis and metastasis. Oncogene. 2018; 37:2660-2675. https://doi. org/10.1038/s41388-018-0162-y. [PubMed]

54. Jiang T, Wang C, Zhu Y, Han H. LINC01410 promotes cell proliferation and migration of cholangiocarcinoma through modulating miR-124-3p/SMAD5 axis. J Gene Med. 2020; 22:e3162. https://doi.org/10.1002/jgm.3162. [PubMed]

55. Kuja-Panula J, Kiiltomäki M, Yamashiro T, Rouhiainen A, Rauvala H. AMIGO, a transmembrane protein implicated in axon tract development, defines a novel protein family with leucine-rich repeats. J Cell Biol. 2003; 160:963-973. https://doi.org/10.1083/jcb.200209074. [PubMed]

56. Xia H, Chen J, Shi M, Gao H, Sekar K, Seshachalam VP, Ooi LLPJ, Hui KM. EDIL3 is a novel regulator of epithelialmesenchymal transition controlling early recurrence of hepatocellular carcinoma. J Hepatol. 2015; 63:863-873. https://doi.org/10.1016/j.jhep.2015.05.005. [PubMed]

57. Wang JP, Hielscher A. Fibronectin: how its aberrant expression in tumors may improve therapeutic targeting. J Cancer. 2017; 8:674-682. https://doi.org/10.7150/jca.16901. [PubMed]

58. Ouderkirk JL, Krendel M. Non-muscle myosins in tumor progression, cancer cell invasion and metastasis. Cytoskeleton (Hoboken). 2014; 71:447-463. https://doi. org $/ 10.1002 / \mathrm{cm} .21187$. [PubMed]

59. Weber L, Maßberg D, Becker C, Altmüller J, Ubrig B, Bonatz G, Wölk G, Philippou S, Tannapfel A, Hatt H, Gisselmann G. Olfactory receptors as biomarkers in human breast carcinoma tissues. Front Oncol. 2018; 8:33. https:// doi.org/10.3389/fonc.2018.00033. [PubMed]

60. Ruan H, Li Y, Wang X, Sun B, Fang W, Jiang S, Liang C. CRYAB inhibits migration and invasion of bladder cancer cells through the PI3K/AKT and ERK pathways. Jpn J Clin Oncol. 2020; 50:254-260. https://doi.org/10.1093/jjco/ hyz172. [PubMed]

61. Reitz C, Conrad C, Roszkowski K, Rogers RS, Mayeux R. Effect of genetic variation in LRRTM3 on risk of Alzheimer disease. Arch Neurol. 2012; 69:894-900. https://doi. org/10.1001/archneurol.2011.2463. [PubMed]

62. Wang J, Yu JT, Jiang T, Tan MS, Wang HF, Tan L, Hu N, Sun L, Zhang W, Tan L. Association of LRRTM3 polymorphisms with late-onset Alzheimer's disease in Han Chinese. Exp Gerontol. 2014; 52:18-22. https://doi. org/10.1016/j.exger.2014.01.013. [PubMed]

63. Wimmer K, Zhu XX, Lamb BJ, Kuick R, Ambros PF, Kovar H, Thoraval D, Motyka S, Alberts JR, Hanash SM. Co- amplification of a novel gene, NAG, with the N-myc gene in neuroblastoma. Oncogene. 1999; 18:233-238. https://doi. org/10.1038/sj.onc.1202287. [PubMed]

64. Shi X, Lu L, Jin X, Liu B, Sun X, Lu L, Jiang Y. GRIN3A and MAPT stimulate nerve overgrowth in macrodactyly. Mol Med Rep. 2016; 14:5637-5643. https://doi. org/10.3892/mmr.2016.5923. [PubMed]

65. Dhillon AS, Hagan S, Rath O, Kolch W. MAP kinase signalling pathways in cancer. Oncogene. 2007; 26:32793290. https://doi.org/10.1038/sj.onc.1210421. [PubMed]

66. Jin DH, Lee J, Kim KM, Kim S, Kim DH, Park J. Overexpression of MAPK15 in gastric cancer is associated with copy number gain and contributes to the stability of c-Jun. Oncotarget. 2015; 6:20190-20203. https://doi. org/10.18632/oncotarget.4171. [PubMed]

67. Yokoyama A. RNA polymerase II-dependent transcription initiated by selectivity factor 1: a central mechanism used by MLL fusion proteins in leukemic transformation. Front Genet. 2019; 9:722. https://doi.org/10.3389/ fgene.2018.00722. [PubMed]

68. Best KE, Rankin J. Long-term survival of individuals born with congenital heart disease: a systematic review and meta-analysis. J Am Heart Assoc. 2016; 5:e002846. https:// doi.org/10.1161/JAHA.115.002846. [PubMed]

69. Dubé BP, Dres M. Diaphragm dysfunction: diagnostic approaches and management strategies. J Clin Med. 2016; 5:113. https://doi.org/10.3390/jcm5120113. [PubMed]

70. Friedman DN, Henderson TO. Late effects and survivorship issues in patients with neuroblastoma. Children (Basel). 2018; 5:107. https://doi.org/10.3390/children5080107. [PubMed]

71. Liu Z, Grant CN, Sun L, Miller BA, Spiegelman VS, Wang HG. Expression patterns of immune genes reveal heterogeneous subtypes of high-risk neuroblastoma. Cancers (Basel). 2020; 12:1739. https://doi.org/10.3390/ cancers 12071739. [PubMed]

72. Russo R, Cimmino F, Pezone L, Manna F, Avitabile M, Langella C, Koster J, Casale F, Raia M, Viola G, Fischer M, Iolascon A, Capasso M. Kinome expression profiling of human neuroblastoma tumors identifies potential drug targets for ultra high-risk patients. Carcinogenesis. 2017; 38:1011-1020. $\quad$ https://doi.org/10.1093/carcin/bgx077. [PubMed]

73. Formicola D, Petrosino G, Lasorsa VA, Pignataro P, Cimmino F, Vetrella S, Longo L, Tonini GP, Oberthuer A, Iolascon A, Fischer M, Capasso M. An 18 gene expressionbased score classifier predicts the clinical outcome in stage 4 neuroblastoma. J Transl Med. 2016; 14:142. https://doi. org/10.1186/s12967-016-0896-7. [PubMed]

74. Goldman M, Craft B, Hastie M, Repečka K, Kamath A, McDade F, Rogers D, Brooks AN, Zhu J, Haussler D. The UCSC Xena platform for public and private cancer genomics data visualization and interpretation. bioRxiv. 2019; 326470. https://doi.org/10.1101/326470. 
75. Vivian J, Rao AA, Nothaft FA, Ketchum C, Armstrong J, Novak A, Pfeil J, Narkizian J, Deran AD, MusselmanBrown A, Schmidt H, Amstutz P, Craft B, et al. Toil enables reproducible, open source, big biomedical data analyses. Nat Biotechnol. 2017; 35:314-316. https://doi.org/10.1038/ nbt.3772. [PubMed]

76. Robinson MD, McCarthy DJ, Smyth GK. edgeR: a Bioconductor package for differential expression analysis of digital gene expression data. Bioinformatics. 2010; 26:139-140. https://doi.org/10.1093/bioinformatics/btp616. [PubMed]

77. Love MI, Huber W, Anders S. Moderated estimation of fold change and dispersion for RNA-seq data with DESeq2. Genome Biol. 2014; 15:550. https://doi.org/10.1186/ s13059-014-0550-8. [PubMed]

78. Yu G, Wang LG, Han Y, He QY. clusterProfiler: an $\mathrm{R}$ package for comparing biological themes among gene clusters. OMICS. 2012; 16:284-287. https://doi. org/10.1089/omi.2011.0118. [PubMed]

79. Yu G, Wang LG, Yan GR, He QY. DOSE: an R/ Bioconductor package for disease ontology semantic and enrichment analysis. Bioinformatics. 2015; 31:608-609. https://doi.org/10.1093/bioinformatics/btu684. [PubMed]
80. Pedregosa F, Varoquaux G, Gramfort A, Michel V, Thirion B, Grisel O, Blondel M, Prettenhofer P, Weiss R, Dubourg V, Vanderplas J, Passos A, Cournapeau D. Scikit-learn: Machine Learning in Python. J Mach Learn Res. 2011; 12:2825-2830.

81. Chang CC, Lin CJ. LIBSVM: A library for support vector machines. ACM Trans Intell Syst Technol. 2011; 2:27. https://doi.org/10.1145/1961189.1961199.

82. Huynh-Thu VA, Irrthum A, Wehenkel L, Geurts P. Inferring regulatory networks from expression data using treebased methods. PLoS One. 2010; 5:e12776. https://doi. org/10.1371/journal.pone.0012776. [PubMed]

83. Shannon P, Markiel A, Ozier O, Baliga NS, Wang JT, Ramage D, Amin N, Schwikowski B, Ideker T. Cytoscape: a software environment for integrated models of biomolecular interaction networks. Genome Res. 2003; 13:2498-2504. https://doi.org/10.1101/gr.1239303. [PubMed] 